\title{
Vagus Nerve Stimulation Applied with a Rapid Cycle Has More Profound Influence on Hippocampal Electrophysiology Than a Standard Cycle
}

\author{
Lars E. Larsen ${ }^{1}$ - Wytse J. Wadman ${ }^{2}$ - Daniele Marinazzo ${ }^{3}$ • Pieter van Mierlo ${ }^{1,4}$. \\ Jean Delbeke ${ }^{1} \cdot$ Sofie Daelemans ${ }^{1} \cdot$ Mathieu Sprengers $^{1} \cdot$ Lisa Thyrion $^{1}$. \\ Wouter Van Lysebettens ${ }^{1} \cdot$ Evelien Carrette $^{1} \cdot$ Paul Boon $^{1} \cdot$ Kristl Vonck $^{1} \cdot$ \\ Robrecht Raedt ${ }^{1}$
}

Published online: 21 April 2016

(C) The American Society for Experimental NeuroTherapeutics, Inc. 2016 parameters was found at $300 \mu \mathrm{A}$, beyond which effects saturated. The findings suggest that rapid-cycle VNS produces a more robust outcome than standard cycle VNS and support already existing preclinical evidence that relatively low output currents are sufficient to produce changes in brain physiology and thus likely also therapeutic efficacy.

Keywords Vagusnervestimulation - Stimulation parameters · Dentate gyrus $\cdot$ Evoked potential $\cdot$ EEG $\cdot$ Phase amplitude coupling

\section{Introduction}

Vagus nerve stimulation (VNS) is an adjunctive treatment for pharmacoresistant epilepsy and depression $[1,2]$. Since gaining regulatory approval, VNS has been used to treat $>70,000$ patients worldwide [3]. Despite numerous preclinical and clinical studies and its widespread application in patients, VNS remains associated with unpredictable and limited therapeutic response rates $[1,4]$, constituting a drawback for an invasive treatment.

Although some attempts have been made to determine whether VNS efficacy depends on stimulation parameters [5, 6], the results are generally confounded by design inconsistencies, in addition to the relatively few parameter settings investigated. Guidelines on the individual optimization of stimulation parameters to improve patient outcomes systematically are unavailable owing to the lack of studies systematically investigating doseresponse relationships. Conducting systematic investigations of VNS parameter efficacy in patients is challenging and almost impossible owing to the huge variability in the treated VNS population, the numerous potential stimulation parameter 
combinations, and the typical limitations of seizure counts as an outcome parameter.

The effect of VNS on different electrophysiological phenomena is now increasingly being investigated in order to understand mechanisms of VNS at a neurophysiological level [7-12]. We previously found in rats that rapid-cycle (7 s on, $18 \mathrm{~s}$ off) VNS at $1000 \mu \mathrm{A}$ modulates dentate gyrus fieldevoked potentials (EPs) induced by stimulation of the perforant path, an afferent fiber bundle projecting to the hippocampus [12]. Dentate field EPs consist of a field excitatory postsynaptic potential (fEPSP), reflecting depolarization of dentate granule cells in response to afferent stimulation. Upon reaching the dentate granule cell discharge threshold, the fEPSP is accompanied by a superimposed population spike, which is the summation of the granule cell action potentials [13]. Dentate field EPs allow us to study excitability, which is an important element in epilepsy [14]. In addition, we found that VNS decreases hippocampal EEG power in various frequency bands [12], reflecting reduced magnitude and/or synchrony of both local and global hippocampal activity [15].

In the present study, we investigated hippocampal effects of various VNS parameters by studying effects of VNS on theta-gamma phase amplitude coupling (PAC) in the hippocampal EEG. Theta-gamma PAC has been described both in human and rodent hippocampus [16, 17], and is thought to be the mechanism by which the activity of local hippocampal neurons or small neuronal assemblies (represented by gamma frequency power) is controlled by field potential changes of larger areas (represented by theta frequency phase) [15]. PAC is hypothesized to support memory encoding and retrieval $[17,18]$.

In addition we aimed to investigate the dose-response relationship between various VNS output currents and effects on hippocampal electrophysiology. Based on previous studies showing that output current $<1000 \mu \mathrm{A}$ is sufficient to recruit vagal fibers effectively [19] and reduce cortical excitability [20], we hypothesized that output currents $<1000 \mu \mathrm{A}$ would also be sufficient to modulate hippocampal electrophysiology. In addition, we hypothesized that a rapid cycle would be more effective than a less intense but more clinically used duty cycle.

\section{Methods}

Twenty-two male Sprague-Dawley rats (Harlan Laboratories, Indianapolis, IN, USA) were used in the present study. Temperature and humidity were controlled, and rats were housed on a $12 \mathrm{~h} / 12 \mathrm{~h}$ light/dark cycle. The experiment was conducted after approval by the local animal experimental committee (Ghent University Hospital, Ghent, Belgium, ECD 12/63) and following European Directive 2010/63/EU.

\section{Surgery}

Animals (weighing 300-380 g) were operated under isoflurane anesthesia (5\% for induction, $2 \%$ for maintenance). A cuff electrode was implanted around the left vagus nerve for VNS, a bipolar recording electrode in the hilus of the left dentate gyrus $(3.8 \mathrm{~mm}$ posterior and $1.9 \mathrm{~mm}$ lateral relative to bregma, approximate depth of $3 \mathrm{~mm}$ relative to brain surface), and a bipolar stimulation electrode in the left dorsomedial perforant path $(7-8 \mathrm{~mm}$ posterior and $3.9 \mathrm{~mm}$ lateral relative to bregma, approximate depth of $2.5 \mathrm{~mm}$ relative to brain surface) [13]. The depth of the electrodes was carefully adjusted to evoke a maximal population spike in the dentate gyrus. Bipolar recording electrodes were made from 2 twisted, polyimide-coated, stainless steel wires with a diameter of $70 \mu \mathrm{m}$ (0.9-mm tip separation). Bipolar stimulation electrodes were made from 2 twisted, polyimide-coated, stainless steel wires with a diameter of $125 \mu \mathrm{m}(0.9-\mathrm{mm}$ tip separation). Electrode leads were collected in a custom-made connector, which was fixed to the skull with stainless steel screws and dental acrylic cement. Following surgery, animals recovered for a minimum of 6 weeks and analgesics (buprenorphine $0.03 \mathrm{mg} / \mathrm{kg} /$ day, s.c.) were administered on the 2 days following surgery.

\section{Recording Sessions}

Following recovery, animals were connected to a video/ electroencephalography (EEG) setup with free movement permitted by a commutator. After habituating to the setup for 1 day, a series of recordings sessions was performed in the freely moving animals. Two electrophysiological parameters were assessed: spontaneous hippocampal EEG and field EPs in the dentate gyrus in response to electrical stimulation of the perforant path. All recording sessions consisted of a 1-h baseline and a 2-h VNS/ SHAM period. The data acquisition design was dependent on the VNS duty cycle (Fig. 1). Two types of VNS duty cycles were investigated: a standard VNS duty cycle (30 s on and $300 \mathrm{~s}$ off) and a rapid VNS duty cycle (7 s on, $18 \mathrm{~s}$ off) [12, 21], which is often used to treat patients not responding to the former scheme $[5,22]$. Two standard cycle conditions were included with VNS applied at intensities of $1000 \mu \mathrm{A}$ (S1000) and $250 \mu \mathrm{A}(\mathrm{S} 250)$. Additionally, 5 rapid cycle conditions with varying VNS intensities were included: 0 (RSHAM), $1000 \mu \mathrm{A}$ (R1000), $250 \mu \mathrm{A}$ (R250), $25 \mu \mathrm{A}$ (R25), and $25 \mu \mathrm{A}$ below behavioral threshold (RSUB). The behavioral VNS threshold was determined by ramping up the output current in $25 \mu \mathrm{A}$ increments until the point where rats displayed the first detectable behavioral reaction to the stimulation. The pulse width of the VNS pulses was $250 \mu$ s and the frequency was $30 \mathrm{~Hz}$ in all cases.

In all sessions, data were acquired in the VNS off period. In standard cycle conditions, the 300 -s off period was split into 12 subrecording cycles of $25 \mathrm{~s}$, during which 2 EPs and a 10-s 


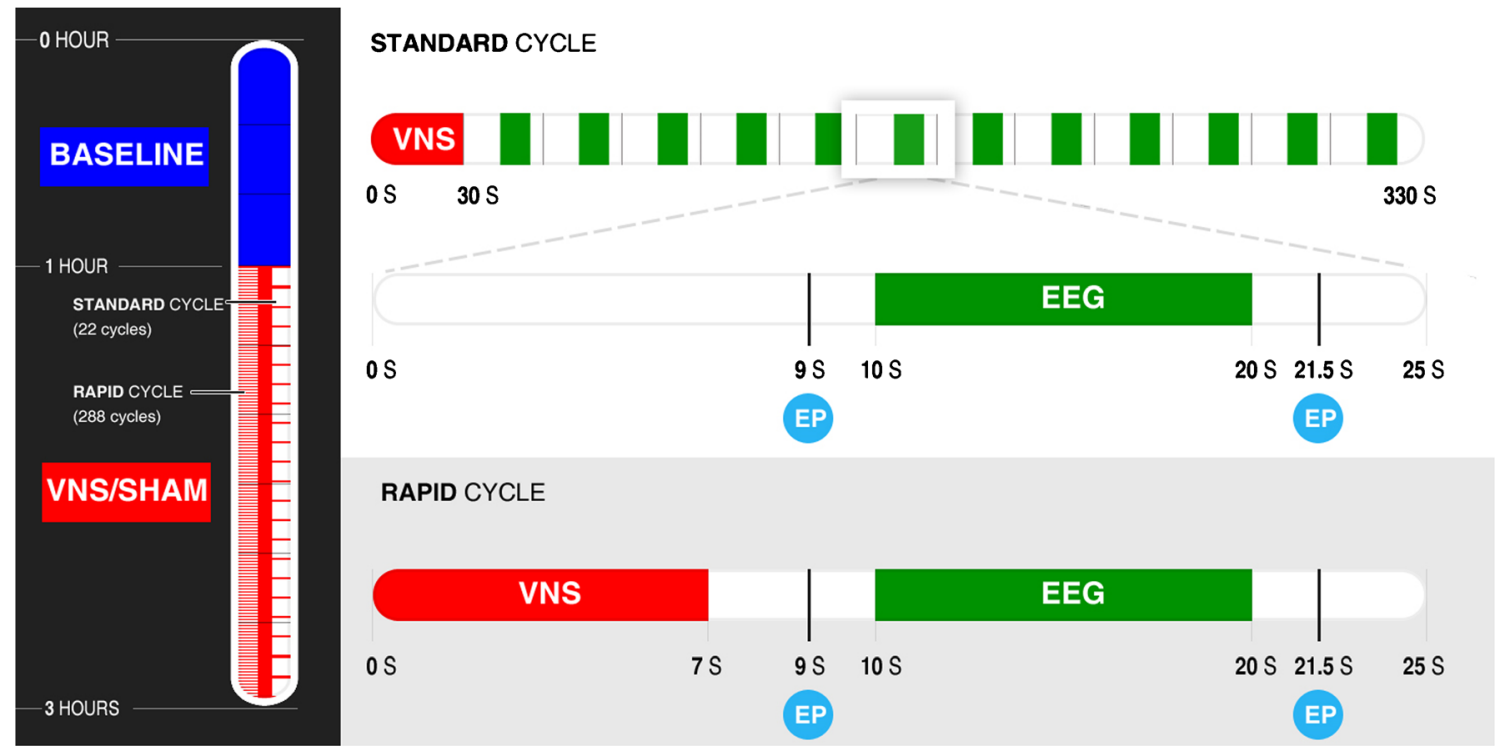

Fig. 1 A schematic illustration of the recording design in all recording conditions. Two conditions followed a standard vagus nerve stimulation (VNS) duty cycle design (VNS on $30 \mathrm{~s}$, off $300 \mathrm{~s}$ ) and 5 conditions followed a rapid duty cycle design (c, VNS on $7 \mathrm{~s}$, off $18 \mathrm{~s}$ ). In all sessions, the 2-h VNS/SHAM period was preceded by a 1-h baseline acquisition period. In the rapid-cycle condition, each duty cycle allowed the acquisition of 2 perforant path-evoked dentate field potentials (EPs)

sweep of EEG were acquired (Fig. 1). This resulted in the acquisition of 24 dentate field EPs and 12 sweeps of EEG between each VNS train. The rapid-cycle recording design resembled the subrecording cycle of the standard-cycle condition, allowing the acquisition of 2 dentate field EPs and a 10-s EEG sweep during each off period of the VNS duty cycle (Fig. 1). In all sessions, the same cycles for data acquisition were used for both baseline and VNS/SHAM periods, though no VNS was applied during baseline. All dentate field EPs were obtained by delivering biphasic pulses to the perforant path with a pulse width of $100 \mu$ s at an intensity adjusted to evoke a dentate population spike of $75 \%$ of maximal amplitude based on an input/output relationship generated prior to the recording sessions.

To establish a dose-response relationship between VNS intensity and changes in hippocampal EEG parameters, an additional experiment was carried out in a subset of 6 rats. On consecutive days, rapid cycle recording sessions were performed using VNS intensities ramped up in daily $25 \mu \mathrm{A}$ increments from 0 to $250 \mu \mathrm{A}$ (day 1-11), in daily increments of $50 \mu \mathrm{A}$ from $300 \mu \mathrm{A}$ to $500 \mu \mathrm{A}$ (day 12-16), and up to $1000 \mu \mathrm{A}$ in a final recording session (day 17). In this experiment, only hippocampal EEG was acquired.

Impedances of stimulation electrodes (both for VNS and perforant path stimulation) were assessed before all sessions. Dentate field EPs and hippocampal EEG were acquired referencing each hippocampal recording electrode to an epidural ground electrode placed above the right frontal cortex. Dentate field EPs were sampled at $20 \mathrm{kHz}$ and hippocampal EEG was and a $10-$ s sweep of spontaneous hippocampal field electroencephalography (EEG) during the off period of the cycle. During the standard cycle condition, the off period was split into 12 subrecording cycles of the same length as the rapid cycle, and, correspondingly, 2 EPs and a 10-s sweep of EEG were recorded. The recording cycle during the baseline period was identical to the VNS/ SHAM period with exception of no VNS being applied

sampled at $1 \mathrm{kHz}$. All signals were high pass filtered at $0.1 \mathrm{~Hz}$ and amplified 100 times, sampling with a 16-bit dynamic and a $\pm 3.05-\mu \mathrm{V}$ input amplitude resolution. Digitization was done with a USB-6259 National Instruments acquisition device (National Instruments, Austin, TX, USA).

\section{Analysis of Dentate Field EPs}

EPs were analyzed with Matlab (MathWorks, Inc., Natick, MA, US). Three parameters were extracted from the dentate field EPs: fEPSP slope, population spike amplitude, and population spike latency (Fig. 2a). fEPSP slope was estimated by fitting a line onto the initial positive deflection of the fEPSP prior to population spike onset, using the least square difference method. Population spike amplitude was estimated by fitting a line between the initial positive peak of the fEPSP, prior population spike onset, and the subsequent positive peak of the fEPSP waveform after the population spike. The line was made tangent to the fEPSP waveform and the population spike amplitude was calculated as the vertical distance from the negative peak of the population spike to this line. Population spike latency was defined as the duration from the stimulus artifact to the negative peak of the population spike.

\section{Spectral Analysis of EEG}

Spectral analysis of the hippocampal EEG was done in Matlab. The 2 hippocampal EEG signals were subtracted to focus on local hippocampal activity. EEG sweeps affected by 
a

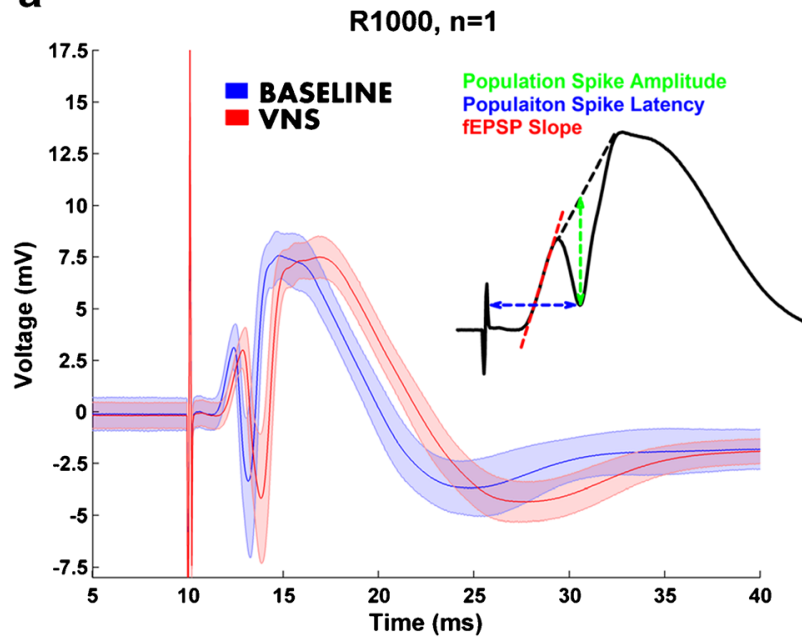

C

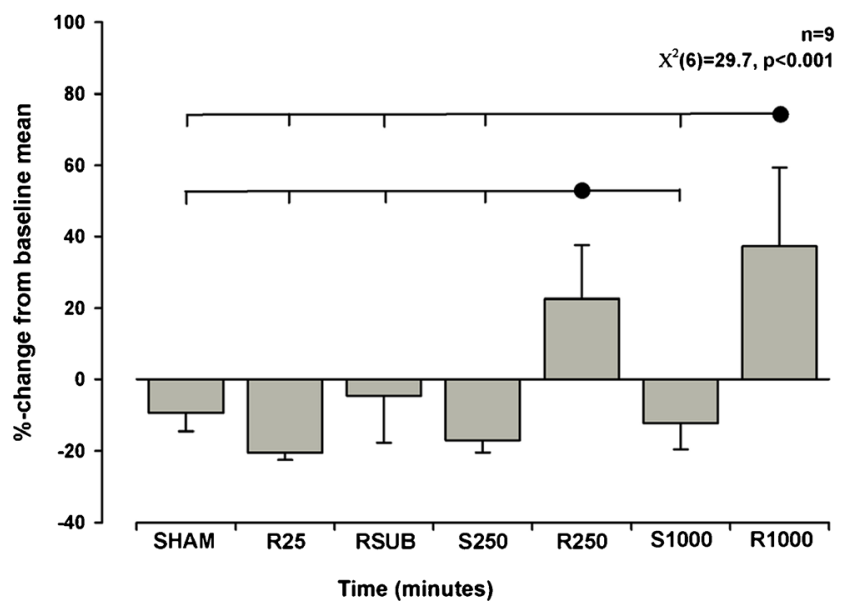

Fig. 2 Effects of vagus nerve stimulation (VNS) on field-evoked potentials (EP) in dentate gyrus in response to electrical stimulation of the perforant path with an intensity that at baseline evoked a population spike of $75 \%$ of the maximum amplitude. In (a), an EP from a representative rat is averaged over the 1-h baseline period (blue) and the last hour of VNS (red) in the rapid cycle $1000 \mu \mathrm{A}$ condition (R1000). The shaded error bars denote the $95 \%$ confidence intervals. The 3 measured outcomes-field excitatory postsynaptic potential (fEPSP) slope, population spike amplitude, and latency — are graphically illustrated in the upper right corner of (a). In (b), (c), and (d), fEPSP slope, population spike amplitude, and latency outcomes have been averaged

artifacts were detected on the basis of total power deviating $>3$ SD from the mean across trials. For power spectral analysis, the EEG traces were subjected to a high pass Butterworth filter at $2 \mathrm{~Hz}$ before splitting the 10-s sweeps into 19 sweeps of $1 \mathrm{~s}$ overlapping by $0.5 \mathrm{~s}$. Using the fast Fourier transform, the 1$100 \mathrm{~Hz}$ power spectrum was extracted at a frequency resolution of $1 \mathrm{~Hz}$. The outcome was 19 power spectra, which were averaged as a representative for each 10-s EEG sweep. Further analysis focused on parameters within the $2-100 \mathrm{~Hz}$ power spectrum. Theta peak frequency was calculated from the mean spectrum of b

fEPSP Slope

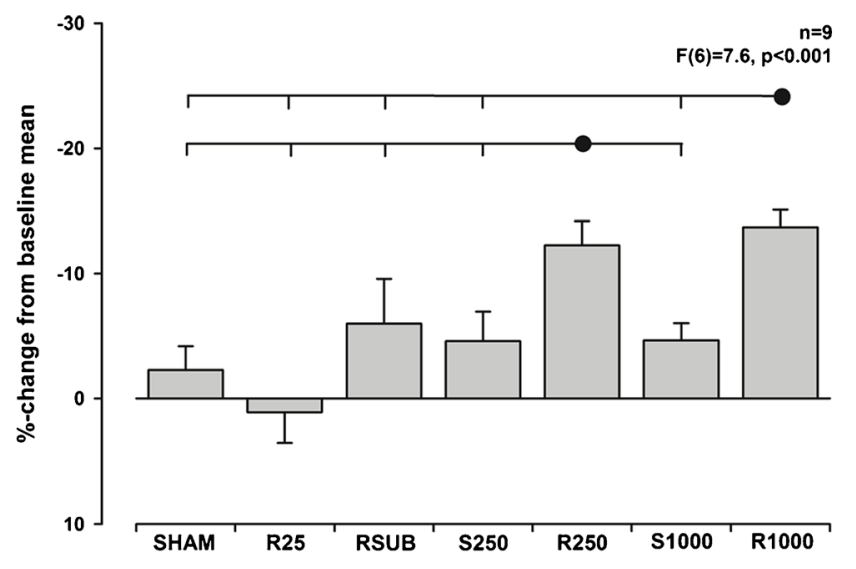

d

Population Spike Latency

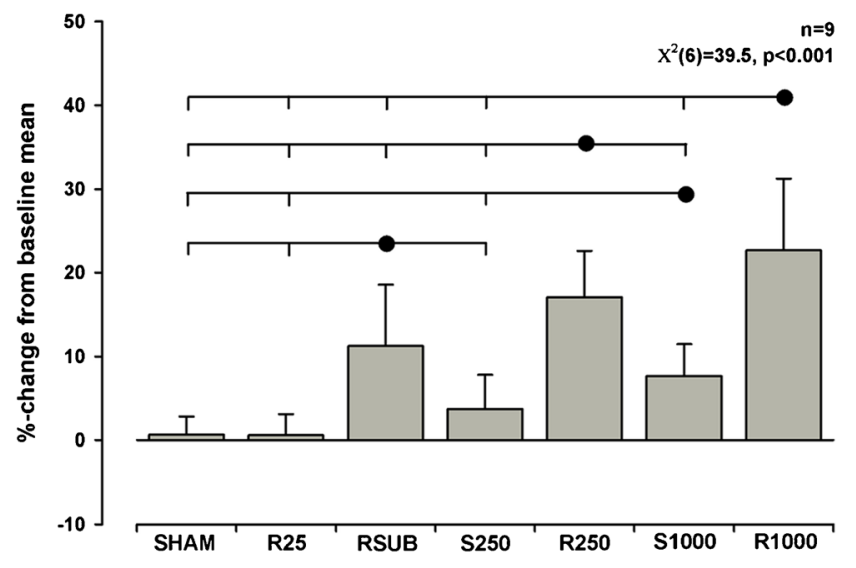

over the last hour of the VNS period for comparison of VNS conditions. The outcome of the 1-way repeated-measures ANOVA is noted in the upper right corner of these plots. The standard cycle (30 s on/300 s off) VNS intensities included were $250 \mu \mathrm{A}$ (S200) and $1000 \mu \mathrm{A}$ (S1000). Further, rapid cycle ( $7 \mathrm{~s}$ on/18 s off) VNS conditions with intensities of $25 \mu \mathrm{A}$ (R25), an intensity below the behavioral threshold (RSUB), $250 \mu \mathrm{A}$ (R250), and $1000 \mu \mathrm{A}$ (R1000) were included. Lines connecting bars denote post-hoc significant differences between the VNS conditions. Bars represent means \pm SEM. Note that for plot (b), the direction of the $y$-axis has been reversed

the last hour of the VNS/SHAM period. Total hippocampal power was calculated for each EEG sweep by integrating the power within the 2-100 Hz spectrum and averaged over the last hour of the VNS/SHAM period subsequently.

\section{PAC Analysis}

PAC analysis was performed with Matlab. To assess PAC in the hippocampal EEG and the effects of VNS on this coupling, we used a normalized modulatory index (MI), 
according to the methods of Tort et al. [16]. The MI reflects the modulation of the amplitude of faster oscillations by the phase of slower oscillations, and has previously been used to characterize coupling of theta and gamma frequencies in the hippocampal region [16, 23, 24]. To calculate the MI, hippocampal EEG was band pass filtered to isolate the frequencies of interest (see below). The phase and amplitude information was then extracted from the respective signals by calculating the phase angle and modulus of the Hilbert transform, respectively, and were used to construct a composite phase amplitude time series. The amplitude information was binned into phase bins of 20 degrees (18 bins) and the amplitude distribution was calculated by calculating the mean amplitude of each bin. The amplitude distribution was normalized by dividing each bin with the sum of all bins and finally the MI is calculated as the deviation (Kullback-Leibler distance) from a uniform distribution. To initially explore coupled frequencies, we computed a comodulogram (see Fig. 4a) pairing the phase of 2 $20 \mathrm{~Hz}$ frequencies to the amplitude of frequencies of 15-200 Hz. Phase frequencies were narrowly band pass filtered ( $1 \mathrm{~Hz}$ width), in steps of $0.5 \mathrm{~Hz}$. The amplitude frequencies were band pass filtered with a filter width of $4 \mathrm{~Hz}$ in steps of $2 \mathrm{~Hz}$. Following identification of coupling between the phase of $4-8 \mathrm{~Hz}$ theta activity and the amplitude of $75-150-\mathrm{Hz}$ gamma activity, band pass filters in these respective frequency ranges were applied to isolate the activity of interest. Analysis focused on coupling strength in the final hour of the VNS/SHAM period. To estimate the significance of the coupling, the MI representative of the final hour of the VNS/SHAM period in the SHAM condition was compared with coupling calculated from shuffled EEG trials (surrogate SHAM) from the same last hour of the SHAM condition. Finally, the MI of the final hour of the VNS/ SHAM period was also calculated from all other VNS conditions, for comparison.

\section{Statistical Analysis}

Hippocampal EEG power and dentate field EP parameters were normalized to the mean of the baseline period. For all extracted outcomes, outliers, defined as measurements deviating $>3$ SD from the mean of a full session, were removed before statistical analysis. Statistical analysis was conducted with R 2.12.1 (R Development Core Team), SPSS 22.0 (IBM, Armonk, NY, USA), or SigmaPlot 11.0 (Systat Software Inc., San Jose, CA, USA). As reported previously [9, 10, 12, 21], preclinical VNS studies tend to yield an ineffectively and an effectively stimulated group for reasons yet to be elucidated. The subset of ineffectively stimulated rats was identified after performing a k-means cluster analysis on the average total hippocampal EEG power change in response to rapid-cycle
VNS at $1000 \mu \mathrm{A}$, as described previously [12]. Further analysis only focused on the cluster of rats responding to rapidcycle VNS at $1000 \mu \mathrm{A}$ with a reduction in hippocampal EEG power. All outcome parameters were averaged over the last hour of the VNS/SHAM period and VNS conditions were compared in a 1-way repeated measures ANOVA. In cases of normality violation, a Friedman ANOVA was applied. The type of outcome statistics, noted in figures or text, denotes the type of test used. Post-hoc evaluation was performed using the Student Newman-Keuls post-hoc test. Statistical significance was considered at $p<0.05$.

Methods related to the creation of specific graphs are explained in the figure legends. All time graphs were created with SigmaPlot. Values are given as mean \pm SEM.

\section{Results}

Fourteen of 22 rats displayed dentate field EPs of adequate and stable quality and were used for the subsequent recordings. K-means cluster analysis revealed that VNS in the R1000 session reduced hippocampal EEG power in 9/14 rats, while no effect was seen in the remaining 5/14 rats. Further analysis focused on the $9 / 14$ rats.

\section{The Effect of Various VNS Parameters on Dentate Field EPs}

VNS was associated with a decrease in fEPSP slope, an increase in population spike amplitude, and an increase in population spike latency (Fig. 2). Comparison of VNS conditions based on the average effect in the last hour of the VNS/SHAM period revealed a main effect of VNS condition on fEPSP slope [Fig. 2b; $\mathrm{F}(6)=7.6 ; p<0.001$ ], population spike amplitude [Fig. 2c; $\chi^{2}(6)=29.7 ; p<0.001$ ], and population spike latency (Fig. 2d; $\chi^{2}(6)=39.5 ; p<0.001$ ]. Relative to SHAM, a significant decrease in fEPSP slope was observed in the R250 and R1000 conditions. Further, decreases in fEPSP slope in R250 and R1000 were significantly more prominent than observed in any of the other conditions, including the standard-cycle conditions with equivalent or higher output currents. Similarly, significant increases in population spike amplitude, relative to SHAM, were only observed in the R250 and R1000 conditions. Compared with SHAM, population spike latency was significantly increased in the RSUB, R250, S1000, and R1000 conditions. Rapid-cycle conditions R250 and R1000 more prominently increased population spike latency than S250 and S1000 conditions. Relative to SHAM, no effect of VNS on any of the measured dentate field EP parameters in the R25 or S250 sessions could be demonstrated. 


\section{The Effect of Various VNS Parameters on Hippocampal EEG}

VNS was associated with a general reduction in broadband 2$100 \mathrm{~Hz}$ hippocampal power, which was most prominent in the theta frequency band $(4-12 \mathrm{~Hz}$ ) and gamma frequency ranges $(>30 \mathrm{~Hz})$, while little change was observed in an intermediate $15-30 \mathrm{~Hz}$ band (Fig. 3a, b). VNS further reduced the theta peak frequency by $2 \mathrm{~Hz}$ (Fig. 3b). Shifting the entire $2-100 \mathrm{~Hz}$ hippocampal power spectrum obtained during VNS towards
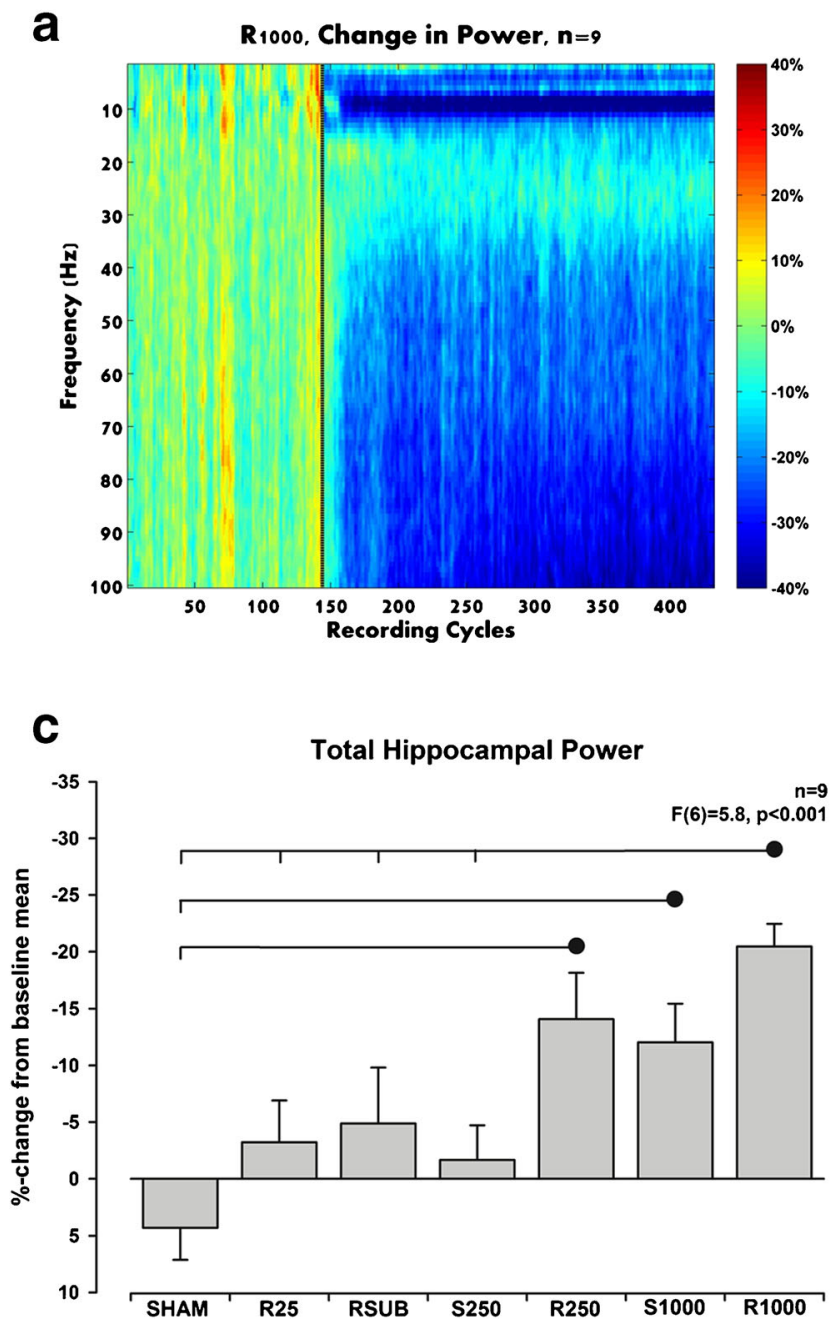

Fig. 3 Effects of vagus nerve stimulation (VNS) on hippocampal electroencephalography. In (a), the normalized change from baseline mean power within $1-\mathrm{Hz}$ frequency bins has been calculated. Warm colors and cold colors represent an increase and decrease in power, respectively. The matrix from which the plot has been created has been subjected to a $3 \times 3$ Gaussian smoothing filter with a sigma of 0.7 prior to creation. In (b), the $2-100-\mathrm{Hz}$ hippocampal power spectrum has been averaged across the 1-h baseline period (blue) and the last hour of the rapid cycle $1000 \mu \mathrm{A}$ VNS condition (red) to demonstrate the change in theta peak frequency. In order to compensate for the shift in frequency observed during VNS, an additional plot has been added where the VNS spectrum has been shifted $+2 \mathrm{~Hz}$ (green), which shows that despite compensation for the shift in frequency, reductions in power during higher frequencies, to compensate for this reduction in theta peak frequency, conserved the above-described decreases in power (Fig. 3b). An effect of stimulation condition was found on hippocampal (2-100 Hz) EEG power [Fig. 3c; $\mathrm{F}(6)=5.8$; $p<0.001]$. Relative to SHAM, a significant decrease in hippocampal EEG power was observed in R250, S1000, and R1000 conditions. The reduction in hippocampal EEG power observed in the R1000 condition was significantly more prominent than observed in the R25, RSUB, and S250 conditions. Stimulation condition also had a significant effect on
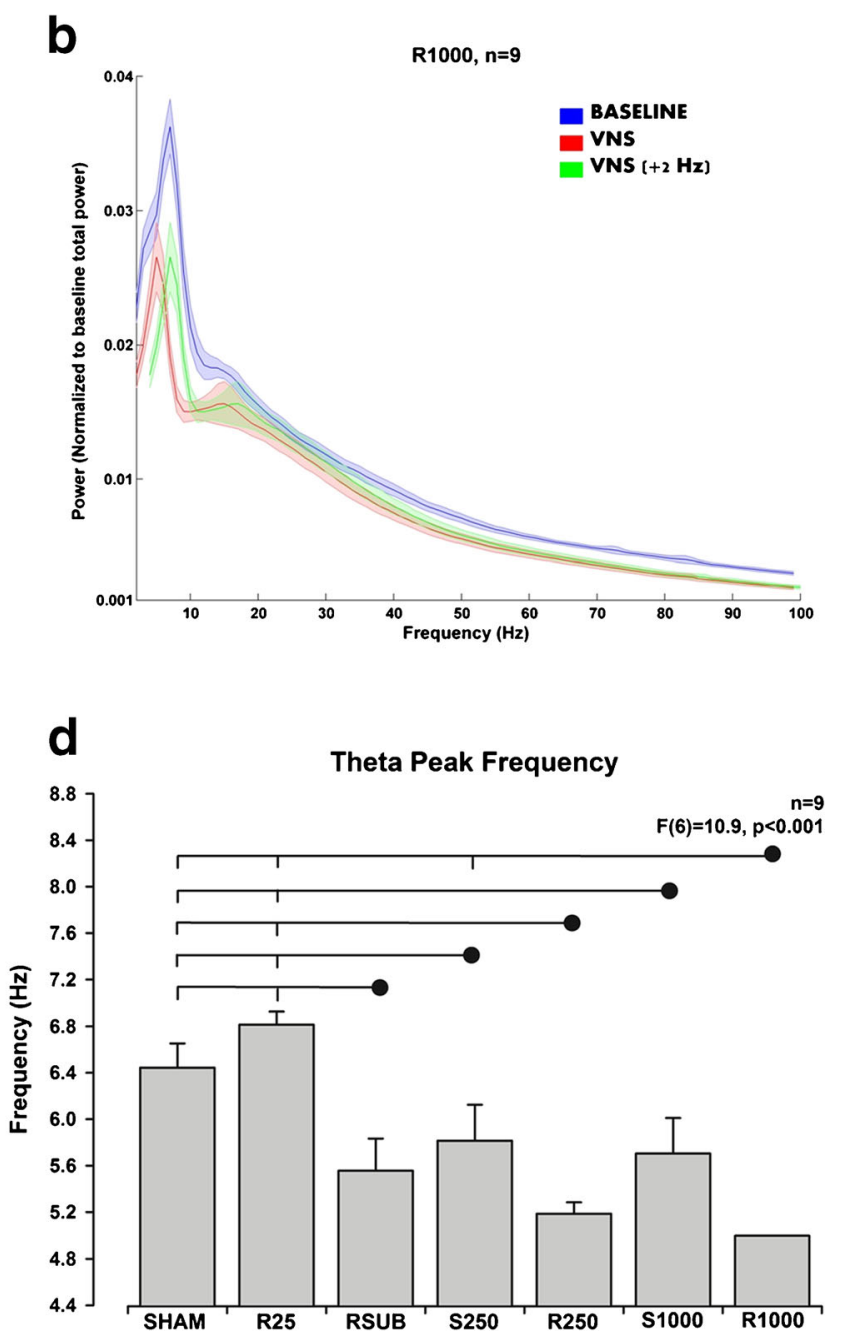

VNS are conserved. The shaded bars represent the $95 \%$ confidence intervals. In (c) and (d), the hippocampal electroencephalography power $(2-100 \mathrm{~Hz})$ and theta peak frequency outcomes have been averaged over the last hour of the VNS period for comparison of VNS conditions. The outcome of the 1-way repeated measures ANOVA is given in the upper right corner of these plots. The standard cycle $(30 \mathrm{~s}$ on/300 s off) VNS intensities included were $250 \mu \mathrm{A}$ (S200) and $1000 \mu \mathrm{A}$ (S1000). Further, rapid cycle (7 s on/18 s off) VNS conditions with intensities of $25 \mu \mathrm{A}(\mathrm{R} 25)$, an intensity below the behavioral threshold (RSUB), $250 \mu \mathrm{A}$ (R250), and $1000 \mu \mathrm{A}$ (R1000) were included. Post-hoc significant differences between VNS conditions are marked with lines between the bars. All bars denote means \pm SEM. Note that for plots (c) and (d) the direction of the $y$-axis has been reversed 
theta peak frequency [Fig. 3d; F(6) $=10.9 ; p<0.001]$. Relative to SHAM and R25 conditions, a significant reduction in theta peak frequency was observed in the RSUB, S250, R250, S1000, and R1000 conditions.

The hippocampal EEG displayed a strong coherence between the phase of theta oscillations in the $4-8-\mathrm{Hz}$ range and the amplitude of fast gamma oscillations in the $75-150 \mathrm{~Hz}$ range (Fig. 4a, b). We found that the power of the fast gamma oscillation was highest at the positive peak of the theta

a

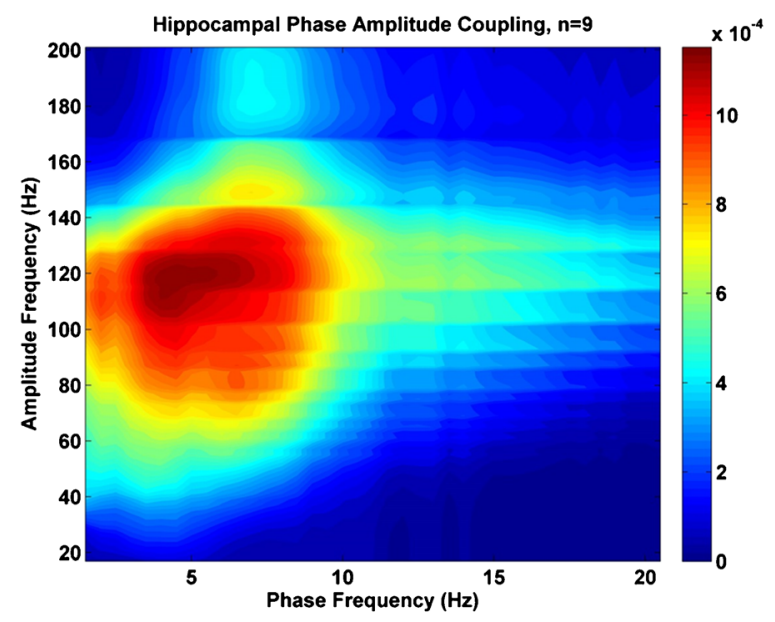

C

Theta (4-8 Hz) Phase, Gamma (75-150 Hz) Amplitude Coupling

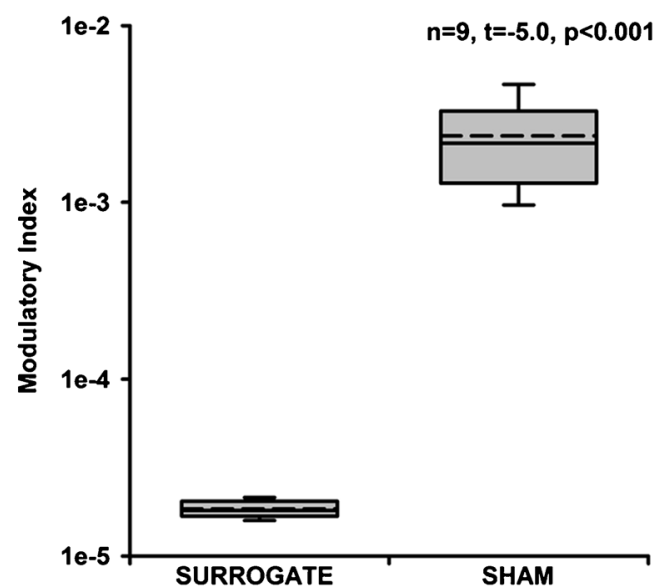

Fig. 4 Phase amplitude coupling between theta and high gamma activity is reduced in response to vagus nerve stimulation (VNS). To explore the coupling between theta and gamma activity in the hippocampal electroencephalography (EEG) initially, a comodulogram based on EEG from the entire SHAM condition was computed (a). The phase frequencies studied were from 2 to $20 \mathrm{~Hz}$ in steps of $0.5 \mathrm{~Hz}$ and were extracted with a band pass filter with a filter width of $1 \mathrm{~Hz}$. The amplitude frequencies were filtered from 15 to $200 \mathrm{~Hz}$ in steps of $2 \mathrm{~Hz}$ and with a filter width of $4 \mathrm{~Hz}$. The amplitude distributions were averaged across rats before calculating a representative modulatory index value for the group for each frequency pairing. Following identification of coupling between 4 and $8 \mathrm{~Hz}$ theta activity and high-frequency oscillations, the connected oscillation. This coupling was well above what can be expected at random, when comparing the data with surrogate data computed by randomly shuffling the data $[$ Fig. $4 \mathrm{c} ; \mathrm{t}(8)=-5$; $p<0.001)$. Compared with SHAM, VNS significantly reduced the theta-gamma PAC but only in the R250 and $\mathrm{R} 1000$ conditions, with the most prominent reduction observed in the R1000 condition. The PAC in the R250 and $\mathrm{R} 1000$ condition was also significantly reduced compared with the other stimulation conditions.

b

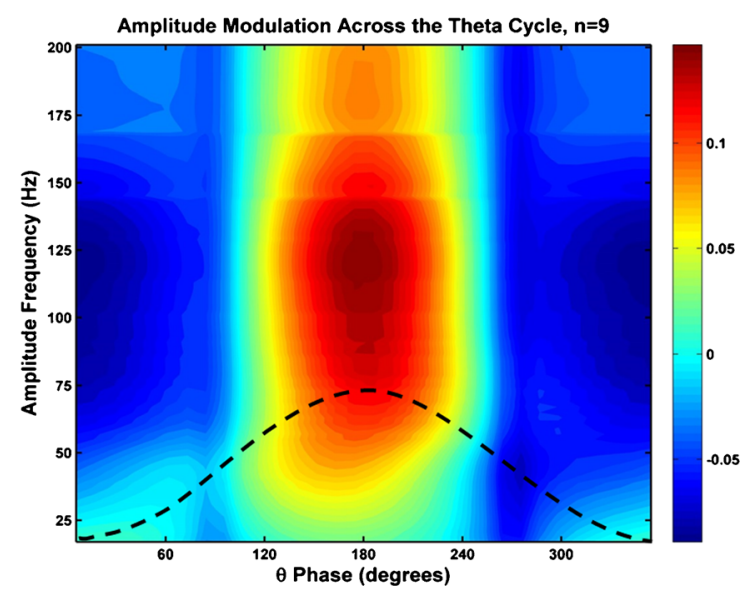

d

Theta (4-8 Hz) - Gamma (75-150 Hz) Coupling during VNS

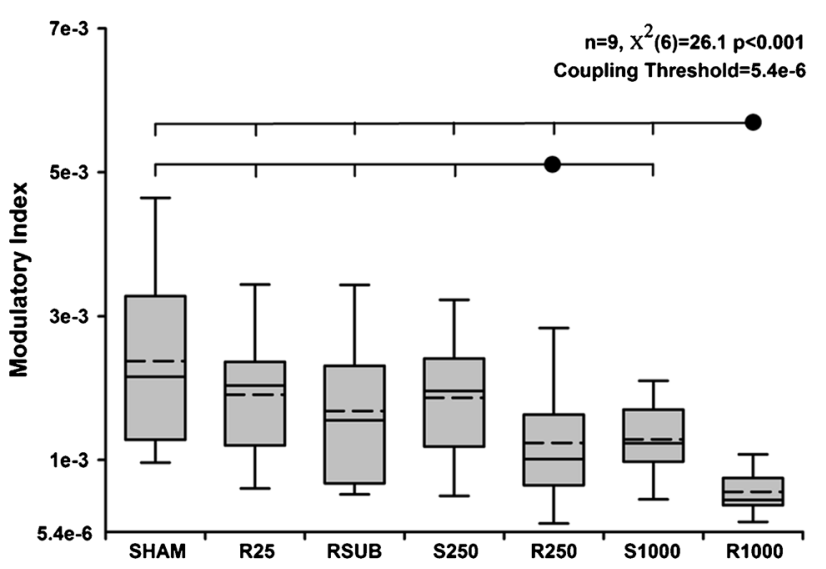

high-frequency components were studied with regard to which phase of the cycle that displayed the largest amplitude (b). Only 1 component in the $75-150 \mathrm{~Hz}$ frequency range was found and used for further analysis. In (c), the robustness of the identified theta-gamma coupling has been studied by comparing the coupling in the SHAM condition to shuffled EEG data from the same condition. Note that in (c), the $y$ axis has a logarithmic scaling. In (d), the modulatory index over the last hour of the VNS period (PERI) has been calculated and compared between VNS conditions in a 1-way repeated measures ANOVA. Post-hoc significant differences between VNS conditions are marked with lines between the bars. Solid lines in the boxplots denote the medians and dashed lines the means 
To more accurately assess the influence of VNS intensity, specifically, on the hippocampal EEG outcomes, a VNS intensity input/output relationship was generated in a subset of 6 rats for the rapid-cycle VNS condition. Stimulation intensity had a significant impact on both hippocampal EEG power $[\mathrm{F}(16)=9.6 ; p<0.001]$, theta peak frequency $[\mathrm{F}(16)=24.6$; $p<0.001]$, and theta-gamma PAC $[\mathrm{F}(16)=5.1 ; p<0.001]$. On average, maximal effect on all parameters was observed around $300 \mu \mathrm{A}$, beyond which further increases in VNS intensity did not yield further effect (Fig. 5).

\section{Discussion}

This study shows that, besides modulating dentate field EPs and reducing hippocampal EEG power and theta peak frequency, VNS also reduces theta-gamma phase amplitude coupling of hippocampal EEG. The present data also demonstrate that the extent by which VNS modulates hippocampal field activity depends on the type of VNS paradigm. Effects observed with rapid-cycle VNS were more pronounced compared with the standard-cycle VNS. Effects of rapid-cycle VNS at $250 \mu \mathrm{A}$ had a greater impact on dentate field EP parameters than standard-cycle VNS at $1000 \mu \mathrm{A}$, while standard-cycle VNS at $250 \mu \mathrm{A}$ induced no change. Finally, effects of rapid-cycle VNS on hippocampal EEG parameters reached maximal levels at output currents of approximately $300 \mu \mathrm{A}$, with no additional effect of increasing the output current.

A reduction in hippocampal EEG power during VNS is in accordance with the early finding of the desynchronizing and power-suppressing effect of vagal afferent stimulation on cortical EEG in cats, though the effect at that time was not quantified with spectral analysis [25]. Owing to the association of a reduction in EEG power and the suppression of strychnineinduced convulsive spike activity [25], EEG desynchronization or reduction in EEG power was believed to reflect the anticonvulsant efficacy of VNS [26]. In the present study, the simultaneous slowing of hippocampal theta rhythm could suggest that a general shift in power towards lower frequencies could produce the general reduction in broadband $2-100 \mathrm{~Hz}$ power found in the present study. However, the observation of reduced power was most pronounced around the theta peak and at gamma frequencies $(>30 \mathrm{~Hz})$, while little effect was observed in the 15 $30-\mathrm{Hz}$ range. If the drop in power during VNS alone should be explained by a shift in the spectrum, one would expect a uniform reduction in power across the whole spectrum, which is not the case. Indeed, when shifting the spectrum to compensate for the slowing observed, reductions in power remained.

The idea that decreased EEG power could reflect a decrease in excitability is supported by the notion that EEG predominantly is an aggregate of synaptic potentials [27]. This may be coupled with the decrease in fEPSP slope of the dentate field EP, which indicates decreased synaptic efficacy. Decreased synaptic efficacy further suggests decreased excitability and could thus explain an anticonvulsive effect. Shorter population spike latencies have further been described in epileptic rats [28], while VNS increased the population spike latency, constituting another effect likely to reflect anticonvulsive efficacy of VNS. The simultaneously observed increase in population spike amplitude, however, contradicts a uniform reduction in excitability during VNS, as it rather indicates the opposite and thus is unlikely to relate to any anticonvulsant effect.

PAC between theta and gamma oscillations has been described previously in different hippocampal regions of the rat $[16,23,24]$. This study shows for the first time a reduced PAC between theta and gamma oscillations during rapid-cycle
Fig. 5 Rapid-cycle vagus nerve stimulation (VNS) output currents were titrated from $25 \mu \mathrm{A}$ to $1000 \mu \mathrm{A}$ in a subset of 6 rats. The figure shows effects of increasing VNS intensities on hippocampal electroencephalography power $(2-100 \mathrm{~Hz})$, theta peak frequency, and theta-gamma coupling. The effect of VNS on the outcomes were averaged over the last hour of the VNS period. Error bars indicate the SEM. Note that the $y$ axis has been reversed to reflect increasing effects of VNS as the output current is increased. $\mathrm{PAC}=$ phase amplitude coupling

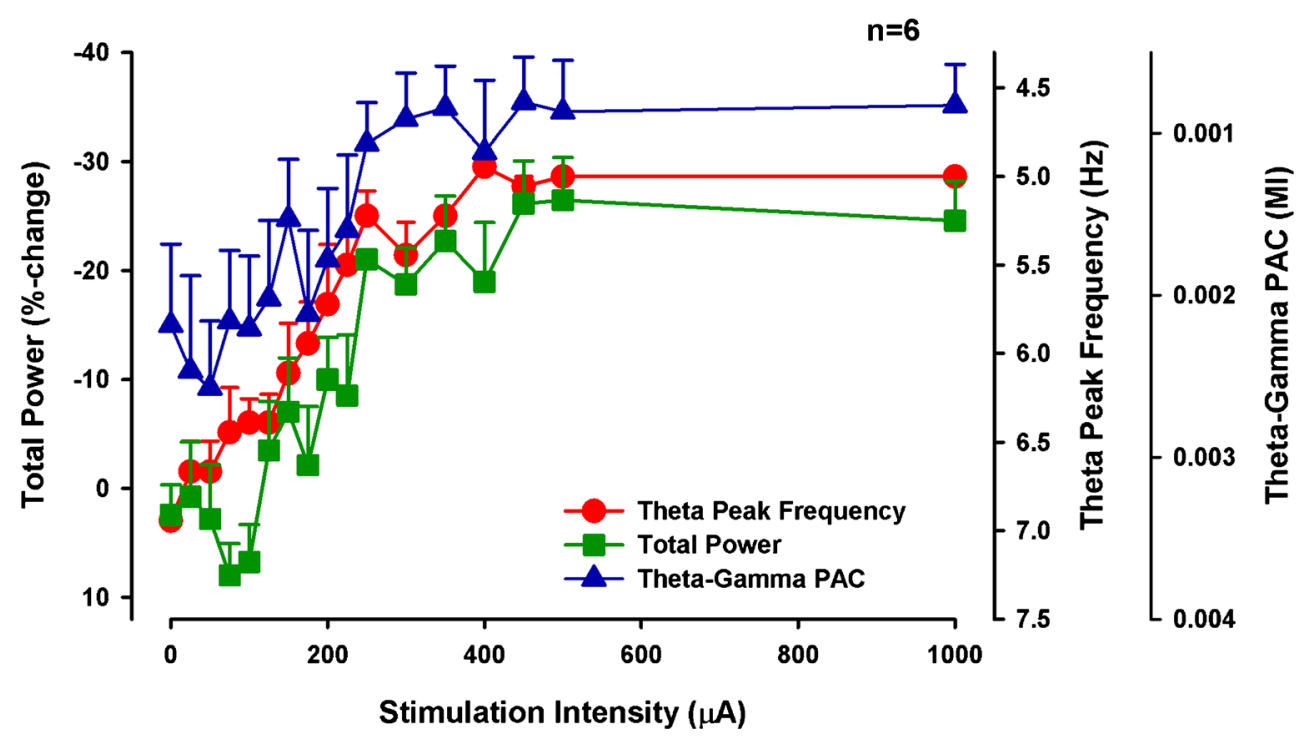


VNS. Although, in theory, the normalized MI is insensitive to absolute changes in power of theta or gamma frequency bands, previous studies reported a correlation between theta power and strength of theta-gamma PAC [16, 23]. This suggests that the amount of theta activity in the hippocampus determines to what degree gamma frequencies are modulated by this theta rhythm. The fact that the coupling strength is reduced during VNS is thus most likely an indirect consequence of the VNS-induced decrease in theta power [29, 30].

Theta-gamma PAC is considered to be supportive for memory encoding and retrieval in the hippocampus $[17,18]$. The reduction of PAC in response to VNS was an unexpected finding, as several studies have reported that VNS can enhance hippocampus-dependent memory and hippocampal long-term potentiation, though mainly in an intermediate output current regimen [11,31-33]. However, none of these studies continuously cycled VNS, as in our study. If theta-gamma PAC does, indeed, play an important role in memory, our findings suggest that continuous cycling of VNS, in particular rapid cycling, might interfere with hippocampus-dependent memory, although such an effect has not yet been reported. However, the reduced theta-gamma PAC in response to rapidcycle VNS could be an important mechanism for suppression of seizure activity. As reduced theta-gamma PAC indicates that local neuronal populations are less recruited by the more global theta rhythm, one could imagine that VNS also prevents their recruitment by pathological seizure rhythms. Although this needs to be proven. However, our data could suggest that VNS-associated anticonvulsant and memoryenhancing effects may require different VNS parameters.

A typical clinical approach to adjustment of VNS parameters involves a continuous adjustment of the stimulator output current just below maximally tolerated levels and a "trial and error" adaptation of the duty cycle, which is typically intensified in poorly responding patients [26]. This concept means that both duty cycle and stimulation intensity are increased, which results in larger amounts of energy requirements, leading to an increased load on the stimulator battery. In the present study, however, it was apparent that lower VNS intensities can already affect brain functioning when using a heavier duty cycle, that is, the rapid cycle. For example, compared with standard-cycle VNS at $1000 \mu \mathrm{A}$, rapid-cycle VNS at $250 \mu \mathrm{A}$ resulted in a similar reduction of EEG power and even more pronounced effects on dentate field EP parameters and theta-gamma PAC in the hippocampal EEG. The active stimulation period of the rapid duty cycle covers $28 \%$ of the time, while the standard cycle, used in the present study, is on for only $9 \%$ of the time. This ratio of $3.1 / 1$ is compensated for by the respective currents of $250 \mu \mathrm{A}$ and $1000 \mu \mathrm{A}$, yielding a power ratio of $1 / 16$ (Watts $=$ Current $^{2} *$ Resistance), and, finally, an energy ratio of 3.1/16 $=0.19$ in favor of the lowcurrent rapid cycle, not to mention the more pronounced effects observed. Electrode safety, biocompatibility, and, especially, battery life of implanted stimulators would be obvious benefits.

A titration of rapid-cycle VNS intensities revealed that reduction of EEG power, slowing of theta rhythm, and reduction in theta-gamma PAC seem to appear at an intensity threshold and then saturate within the range of very few intensity increments. On average, saturation was reached around $300 \mu \mathrm{A}$. This observation aligns with a previous observation from our laboratory that VNS at $250 \mu \mathrm{A}$ was sufficient to reduce cortical excitability [20], while further increases did not yield additional effects. The observation that further increases in stimulation intensity produces no further effects supports the involvement of thick myelinated vagal type A afferents, which generally exhibit a more homogeneous threshold for activation than slowly conducting and less densely myelinated fiber types $[34,35]$. Thick myelinated vagal afferents additionally exhibit a threshold for electrical activation similar to that of efferent motor fibers innervating the laryngeal muscle, and laryngeal muscle-evoked potentials have thus been proposed as a marker for effective recruitment of vagal afferents during VNS [36]. Interestingly, a recent study found a median recruitment threshold for the laryngeal muscle-evoked potential of $300 \mu \mathrm{A}$ [19], which is similar to the intensity where effects of VNS on EEG saturate in the present study. Evidence from dogs further supports that maximal recruitment of thick myelinated vagal afferents is achieved at intensities around $350 \mu \mathrm{A}$ using pulse widths of $300 \mu \mathrm{s}$ [35]. Thus, the presented data strongly support that VNS-associated reduction of EEG power is due to recruitment of thickly myelinated A-fibers and that a saturation of A-fiber recruitment likely reflects a saturation of the observed effects on hippocampal EEG at higher VNS intensities. With no further benefit of increasing the stimulation intensity, this suggests that the current approach to adjustment of VNS parameters should be optimized by using noninvasive measurements to estimate A-fiber recruitment. This finding could further suggest that VNS output current only plays a role in effectively recruiting A-fiber afferents, which implies that beyond this point the "stimulation dose" can only be increased by intensifying the duty cycle, such as applying a rapid cycle instead of a standard cycle. Our findings do, indeed, support that intensification of duty cycle constitutes an option to obtain additional efficacy.

It should be noted, that the present study assessed effects of VNS in the healthy brain, which motivates attempts to replicate results in an epileptic state, to further correlate observed effects to anticonvulsant efficacy of VNS. In relation to this, it is relevant to assess how concomitant antiepileptic drugs influence the outcomes, as this could give information on the possible synergistic effects of antiepileptic drugs and VNS. Additionally, it is noteworthy that the present study only analyzed acute neurophysiological changes during VNS, while the chronic effects could possibly differ or be more pronounced, as it is well known that VNS efficacy tends to 
increase over time [1, 6, 37]. Nevertheless, these results stress the importance of re-evaluating the current approach to VNS parameter selection. Using neurophysiological parameters, as in the present study, it may be possible to develop sophisticated algorithms to adapt VNS parameters to a specific desired clinical outcome for given individual patients.

Acknowledgments Lars Emil Larsen is supported by a grant from the "Bijzonder Onderzoeksfond (BOF)". Professor Robrecht Raedt and Professor Kristl Vonck are supported by grants from the BOF and Ghent University. Professor Paul Boon is supported by grants from the "Fonds Wetenschappelijk Onderzoek (FWO)", the BOF, and the Clinical Epilepsy Grant from Ghent University Hospital. The authors are thankful to Professor Adriano Tort for sharing Matlab codes used to analyze phase amplitude coupling in the hippocampal electroencephalography.

\section{Compliance with Ethical Standards}

Required Author Forms Disclosure forms provided by the authors are available with the online version of this article.

\section{References}

1. Ben-Menachem E. Vagus-nerve stimulation for the treatment of epilepsy. Lancet Neurol 2002; 1: 477-482.

2. Schlaepfer TE, Frick C, Zobel A, et al. Vagus nerve stimulation for depression: efficacy and safety in a European study. Psychol Med 2008; 38: 651-661.

3. Cyberonics ${ }^{\circledR}$. Cyberonics Images and Captions. 2013; Available from: http://www.cyberonics.com/sites/default/files/CyberonicsImages-and-Captions-FINAL-2013.pdf. Accessed 2016 February.

4. Rush AJ, Marangell LB, Sackeim HA, et al. Vagus nerve stimulation for treatment-resistant depression: a randomized, controlled acute phase trial. Biol Psychiatry 2005; 58: 347-354.

5. Labar D. Vagus nerve stimulation for 1 year in 269 patients on unchanged antiepileptic drugs. Seizure 2004; 13: 392398.

6. DeGiorgio CM, Thompson J, Lewis $\mathrm{P}$, et al. Vagus nerve stimulation: analysis of device parameters in 154 patients during the longterm XE5 study. Epilepsia 2001; 42: 1017-1020.

7. Ura H, Sugaya $\mathrm{Y}$, Ohata $\mathrm{H}$, et al. Vagus nerve stimulation induced long-lasting enhancement of synaptic transmission and decreased granule cell discharge in the hippocampal dentate gyrus of urethane-anesthetized rats. Brain Res 2013; 1492: 63-71.

8. Shen H, Fuchino Y, Miyamoto D, Nomura H, Matsuki N. Vagus nerve stimulation enhances perforant path-CA3 synaptic transmission via the activation of beta-adrenergic receptors and the locus coeruleus. Int $\mathrm{J}$ Neuropsychopharmacol 2012; 15: 523-530.

9. Engineer ND, Riley JR, Seale JD, et al. Reversing pathological neural activity using targeted plasticity. Nature 2011; 470: 101-104.

10. Nichols JA, Nichols AR, Smirnakis SM, et al. Vagus nerve stimulation modulates cortical synchrony and excitability through the activation of muscarinic receptors. Neuroscience 2011; 189: 207214.

11. Zuo Y, Smith DC, Jensen RA. Vagus nerve stimulation potentiates hippocampal LTP in freely-moving rats. Physiol Behav 2007; 90: 583-589.
12. Larsen LE, Wadman WJ, van Mierlo P, et al. Modulation of hippocampal activity by vagus nerve stimulation in freely moving rats. Brain Stimul 2016; 9: 124-132.

13. McNaughton BL and Barnes CA. Physiological identification and analysis of dentate granule cell responses to stimulation of the medial and lateral perforant pathways in the rat. J Comp Neurol 1977; 175: 439-454.

14. Prince DA, Connors BW, Benardo LS. Mechanisms underlying interictal-ictal transitions. Adv Neurol 1983; 34: 177-187.

15. Lisman JE and Jensen O. The theta-gamma neural code. Neuron 2013; 77: 1002-1016.

16. Tort AB, Komorowski R, Eichenbaum H, Kopell N. Measuring phase-amplitude coupling between neuronal oscillations of different frequencies. J Neurophysiol 2010; 104: 1195-1210.

17. Axmacher N, Henseler MM, Jensen O, et al. Crossfrequency coupling supports multi-item working memory in the human hippocampus. Proc Natl Acad Sci U S A 2010; 107: 3228-3233.

18. Park JY, Lee YR, Lee J. The relationship between theta-gamma coupling and spatial memory ability in older adults. Neurosci Lett 2011; 498: 37-41.

19. Grimonprez A, Raedt R, De Taeye L, et al. A preclinical study of laryngeal motor-evoked potentials as a marker vagus nerve activation. Int J Neural Syst 2015;25:1550034.

20. Mollet L, Grimonprez A, Raedt R, et al. Intensity-dependent modulatory effects of vagus nerve stimulation on cortical excitability. Acta Neurol Scand 2013; 128: 391-396.

21. Raedt R, Clinckers R, Mollet L, et al. Increased hippocampal noradrenaline is a biomarker for efficacy of vagus nerve stimulation in a limbic seizure model. J Neurochem 2011; 117: 461-469.

22. Liporace J, Hucko D, Morrow R, et al. Vagal nerve stimulation: adjustments to reduce painful side effects. Neurology 2001; 57: 885-886.

23. Tort AB, Scheffer-Teixeira R, Souza BC, Draguhn A, Brankack J. Theta-associated high-frequency oscillations $(110-160 \mathrm{~Hz})$ in the hippocampus and neocortex. Prog Neurobiol 2013; 100: 1-14.

24. Bott JB, Muller MA, Jackson J, et al. Spatial reference memory is associated with modulation of theta-gamma coupling in the dentate gyrus. Cereb Cortex 2015 Aug 6.

25. Zanchetti A, Wang SC, Moruzzi G. The effect of vagal afferent stimulation on the EEG pattern of the cat. Electroencephalogr Clin Neurophysiol 1952; 4: 357-361.

26. Heck C, Helmers SL, DeGiorgio CM. Vagus nerve stimulation therapy, epilepsy, and device parameters: scientific basis and recommendations for use. Neurology 2002; 59: S31-S37.

27. Buzsaki G, Anastassiou CA, Koch C. The origin of extracellular fields and currents-EEG, ECoG, LFP and spikes. Nat Rev Neurosci 2012; 13: 407-420.

28. Queiroz CM, Gorter JA, Lopes da Silva FH, Wadman WJ. Dynamics of evoked local field potentials in the hippocampus of epileptic rats with spontaneous seizures. J Neurophysiol 2009; 101: $1588-1597$.

29. Tort AB, Kramer MA, Thorn C, et al. Dynamic cross-frequency couplings of local field potential oscillations in rat striatum and hippocampus during performance of a T-maze task. Proc Natl Acad Sci U S A 2008; 105: 20517-20522.

30. Scheffer-Teixeira R, Belchior H, Caixeta FV, et al. Theta phase modulates multiple layer-specific oscillations in the CA1 region. Cereb Cortex 2012; 22: 2404-2414.

31. Clark KB, Naritoku DK, Smith DC, Browning RA, Jensen RA. Enhanced recognition memory following vagus nerve stimulation in human subjects. Nat Neurosci 1999; 2: 94-98. 
32. Clark KB, Krahl SE, Smith DC, Jensen RA. Post-training unilateral vagal stimulation enhances retention performance in the rat. Neurobiol Learn Mem 1995; 63: 213-216.

33. Clark KB, Smith DC, Hassert DL, et al. Posttraining electrical stimulation of vagal afferents with concomitant vagal efferent inactivation enhances memory storage processes in the rat. Neurobiol Learn Mem 1998; 70: 364-373.

34. Krahl SE, Senanayake SS, Handforth A. Destruction of peripheral $\mathrm{C}$-fibers does not alter subsequent vagus nerve stimulation-induced seizure suppression in rats. Epilepsia 2001; 42: 586-589.
35. Yoo PB, Lubock NB, Hincapie JG, et al. High-resolution measurement of electrically-evoked vagus nerve activity in the anesthetized dog. J Neural Eng 2013; 10: 026003.

36. Mollet L, Raedt R, Delbeke J, et al. Electrophysiological responses from vagus nerve stimulation in rats. Int J Neural Syst 2013; 23 : 1350027.

37. DeGiorgio CM, Schachter SC, Handforth A, et al. Prospective long-term study of vagus nerve stimulation for the treatment of refractory seizures. Epilepsia 2000; 41: 1195-1200. 